\title{
The Chinese (Cantonese) Montreal Cognitive Assessment in Patients with Subcortical Ischemic Vascular Dementia
}

\author{
Jin-song You Ri-zhao Chen Fo-ming Zhang Zi-yi Zhou \\ Ye-feng Cai Gui-fu Li
}

Department of Neurology, Second Affiliated Hospital, Guangzhou University of Traditional

Chinese Medicine, Guangzhou, People's Republic of China

\section{Key Words}

Dementia $\cdot$ Cognitive impairments $\cdot$ Montreal Cognitive Assessment $\cdot$ Neuropsychology

Vascular dementia

\begin{abstract}
Background: Subcortical ischemic vascular dementia (SIVD) has been proposed as the most frequent subtype of vascular cognitive impairment. The aim of this study was to evaluate the psychometric properties of the Chinese (Cantonese) Montreal Cognitive Assessment (CCMoCA) in patients with SIVD in the Guangdong Province of China. Methods: 71 SIVD patients and 60 matched controls were recruited for the CC-MoCA, Mini Mental State Examination and executive clock drawing tasks. Receiver-operating characteristic curve analyses were performed to determine optimal sensitivity and specificity of the CC-MoCA total score in differentiating mild vascular dementia $(\mathrm{VaD})$ patients from moderate $\mathrm{VaD}$ patients and controls. Results: The mean CC-MoCA scores of the controls, and mild and moderate VaD patients were $25.2 \pm 3.8$, $16.4 \pm 3.7$, and $10.0 \pm 5.1$, respectively. In our study, the optimal cutoff value for the CC-MoCA to be able to differentiate patients with mild VaD from controls is $21 / 22$, and $13 / 14$ to differentiate mild VaD from moderate VaD. Conclusion: The CC-MoCA is a useful cognitive screening instrument in SIVD patients.

Copyright $\odot 2011$ S. Karger AG, Basel
\end{abstract}

This study was supported by the Natural Science Foundation of Guangdong (No. 10151040701000064). 


\section{Introduction}

Subcortical ischemic vascular dementia (SIVD) has been proposed as the most frequent subtype of vascular cognitive impairment (VCI) [1]. The syndrome is defined clinically by cognitive impairment and evidence of subcortical vascular brain injury, including lacunar infarcts and deep white matter changes. SIVD has been traditionally recognized as lacunar state, strategic infarct dementia and Binswanger syndrome [2]. Executive dysfunction, which is characterized by decreasing mental processing speed, decreased working memory, and impaired abstract reasoning, is a recognized feature of VCI. Cognitive tests can usefully differentiate between vascular dementia (VaD) and Alzheimer's disease (AD), but standard cognitive assessments, such as the Mini Mental State Examination (MMSE) [3], are insensitive to detect patients with mild symptoms and non-Alzheimer's type cognitive impairment such as VCI [4]. Recently, the Montreal Cognitive Assessment (MoCA) [5] has been developed in view of the inadequacies of the MMSE. It is recommended by the National Institute of Neurological Disorders and Stroke-Canadian Stroke Network VCI Harmonization Standard as a brief cognitive tool for the early detection of VCI [6]. Unlike the MMSE, the MoCA includes additional memory items and emphasizes attentional/executive and language tasks. The validity of the MoCA has been studied in various clinical groups [7-11]. Using a cutoff score $\leq 26$, the sensitivity of the MoCA for identifying mild cognitive impairment (MCI) and AD was excellent (90 and $100 \%$, respectively). Specificity for correctly classifying cognitively normal individuals was also very high (87\%) [5]. A follow-up study using a prospective design conducted in the UK found similarly high sensitivities of 83 and 94\%, respectively, for MCI and dementia of any kind. Specificity however was modest (50\%) [12]. Recently, using a translated version of the MoCA in a study conducted in Korea, Lee et al. [13] reported high sensitivities for the detection of MCI using lower cutoff scores of 22/23. Most of these studies only examined the ability of the MoCA to differentiate between diagnostic groups (i.e. criterion validity) but did not reflect the degree of cognitive injury in VCI. Moreover, in patients with mild-to-moderate $\mathrm{VaD}$, the score area and cutoff value of the MoCA has not been reported. The current study intended to investigate the score area of the Chinese (Cantonese) MoCA (CC-MoCA) in SIVD patients and cognitively normal healthy controls in the Guangdong Province of China.

\section{Patients and Methods}

\section{Subjects}

Patients were recruited from the Stroke Center of the Second Affiliated Hospital of the Guangzhou University of Traditional Chinese Medicine. The inclusion criteria were (1) a diagnosis of possible or probable VaD lasting $>3$ months, as defined by NINDS-AIREN criteria; (2) MRI evidence of significant SIVD, which is defined as the presence of lacunar state, strategic infarct, and confluent white matter lesions (WMLs); (3) mild and moderate dementia [global score of 1 and 2 by Clinical Dementia Rating (CDR), respectively] [14]; (4) an MMSE score between 10 and 26, and (5) ability to perform cognitive and motor tasks for neuropsychological assessment. Patients with significant depression or other psychiatric disorders assessed by the 4th edition of the Diagnostic and Statistical Manual of Mental Disorders [15] and MRI evidence of cortical infarct or a history of hemorrhagic stroke were excluded. Controls were relatives of the study patients, staff or volunteers recruited from the hospital or nearby community centers. Inclusion criteria for controls were (1) normal cognition defined by a CDR of 0 and MMSE $\geq 26$ and (2) absence of a history of stroke. Subjects were also excluded from participating as a control if they had evidence of significant small vascular disease on MRI or cognitive complaints. Common exclusion criteria for all subjects 
were (1) presence of coexisting medical conditions potentially affecting cognition (e.g. vitamin $\mathrm{B}_{12}$ deficiency, hypothyroidism, chronic alcoholism, and active psychiatric disorders); (2) other conditions that might affect testing (e.g. severe aphasia, visual or hearing impairment), and (3) contraindication to MRI. All subjects were of Chinese ethnicity and spoke Cantonese as their primary language. The study was approved by the Clinical Research Ethics Committee of the Guangzhou University of Traditional Chinese Medicine, and written informed consent was given by each participant.

\section{MRI Examination}

MRI was performed on all subjects using a 1.5-tesla scanner (Sonata; Siemens Medical Systems, Erlangen, Germany). WMLs were determined as ill-defined hyperintensities with a diameter $\geq 5 \mathrm{~mm}$ on $\mathrm{T}_{2}$ and fluid-attenuated inversion recovery sequences. A neurologist and a trained scientist visually assessed the severity of WMLs on these sequences, graded WMLs in 5 areas (frontal, parietal-occipital, temporal, infratentorial, and basal ganglia) using the scale of Wahlund et al. [16] and further grouped the lesions as nonconfluent (grades 0-2) or confluent (grade 3). Significant SIVD is defined as the presence of a confluent lesion in $\geq 1$ area.

\section{Scale Assessment}

The MMSE, CC-MoCA and executive clock drawing task (CLOX) tests were administered approximately 3 months after discharge to the stroke patients. The Chinese MMSE [17] was used to index general cognition. The CC-MoCA form and administration instructions are available for download at the MoCA official website (http://www.mocatest.org). Scores of the MMSE and MoCA range from 0 to 30. The CLOX has been divided into two parts to help discriminate the executive control of clock drawing from clock drawing itself [18]. The patient is first instructed to draw a clock on the back of the CLOX form. The instructions can be repeated until they are clearly understood, but once the subject begins to draw no further assistance is allowed. The subject's performance is rated according to the CLOX directions, and scored as 'CLOX1'. The second step of CLOX is a simple copying task. The examiner allows the patient to observe him or her drawing a clock in the circle provided on the scoring sheet. The patient is allowed to copy the examiner's clock. This clock is scored as 'CLOX2'. Scores of the CLOX1 and CLOX2 range from 0 to 15.

\section{Analyses}

All statistical procedures were performed with SPSS 15.0 for Windows (SPSS Inc., Chicago, Ill., USA). Demographic and neuropsychological data are compared between controls and patients using an independent $t$ test or the $\chi^{2}$ test, as appropriate. The MMSE, CCMoCA, CLOX1 and CLOX2 scores were compared with age, gender, and education adjusted using analysis of covariance.

We examined the ability of the CC-MoCA total score in differentiating mild VaD patients from controls and moderate $\mathrm{VaD}$ patients using a receiver operating curve (ROC) analysis. We then derived a cutoff score at the optimal balance of sensitivity and specificity.

\section{Results}

\section{Subject Characteristics}

Seventy patients and 61 controls were recruited. Thirty $(n=30,43 \%)$ of the patients were rated as having mild dementia (CDR 1$)$, while the remainder $(\mathrm{n}=40,57 \%)$ was rated as having moderate dementia (CDR 2). The mean age and duration of education for the total sample were $70.1(\mathrm{SD}=7.88)$ and 8.84 years $(\mathrm{SD}=3.20)$, respectively. Demographic information, 
Table 1. Demographic data and clinical features of the study subjects

\begin{tabular}{lcccc}
\hline & $\begin{array}{l}\text { Controls } \\
(\mathrm{n}=61)\end{array}$ & $\begin{array}{l}\text { Mild VaD } \\
(\mathrm{n}=30)\end{array}$ & $\begin{array}{l}\text { Moderate VaD } \\
(\mathrm{n}=40)\end{array}$ & $\mathrm{p}$ value \\
\hline Age, years & $68.8 \pm 7.4$ & $70.1 \pm 8.4$ & $71.7 \pm 8.1$ & 0.187 \\
Education, years & $9.3 \pm 3.3$ & $9.7 \pm 3.2$ & $7.5 \pm 2.5^{\mathrm{a}}$ & 0.005 \\
Females, \% & 67.4 & 46.2 & 64.4 & 0.066 \\
MMSE & $28.3 \pm 1.5^{\mathrm{b}}$ & $22.2 \pm 1.4^{\mathrm{b}}$ & $15.4 \pm 2.9^{\mathrm{b}}$ & $<0.001$ \\
MoCA & $25.2 \pm 3.8^{\mathrm{b}}$ & $16.4 \pm 3.7^{\mathrm{b}}$ & $10.0 \pm 5.1^{\mathrm{b}}$ & $<0.001$ \\
CLOX1 & $12.4 \pm 1.49^{\mathrm{b}}$ & $8.9 \pm 3.4^{\mathrm{b}}$ & $5.8 \pm 4.1^{\mathrm{b}}$ & $<0.001$ \\
CLOX2 & $13.8 \pm 1.2^{\mathrm{b}}$ & $12.1 \pm 2.4^{\mathrm{b}}$ & $8.6 \pm 3.9^{\mathrm{b}}$ & $<0.001$ \\
\hline
\end{tabular}

Means (crude) \pm SD.

${ }^{\mathrm{a}}$ Moderate $\mathrm{VaD}<$ mild $\mathrm{VaD}$ and controls $(\mathrm{p}=0.005)$.

${ }^{\mathrm{b}}$ Moderate $\mathrm{VaD}<$ mild $\mathrm{VaD}<$ controls $(\mathrm{p}<0.001)$.

and MMSE, MoCA and CLOX scores for the three groups are shown in table 1. No differences were found among groups regarding age $(\mathrm{F}=1.697, \mathrm{p}=0.187)$ and gender $\left(\chi^{2}=5.442\right.$, $\mathrm{p}=0.066)$. The educational level of patients with moderate $\mathrm{VaD}$ was lower than that of $\mathrm{pa}-$ tients with mild $\mathrm{VaD}(\mathrm{p}=0.004)$ and controls $(\mathrm{p}=0.007)$.

\section{Group Differences and ROC Analysis of MoCA, CLOX and MMSE Performance}

The mean MMSE and MoCA scores of the controls were $28.3 \pm 1.5$ and $25.2 \pm 3.8$, respectively. Although normal MMSE scores $(\geq 26)$ were part of the eligibility criteria, $45.3 \%$ of the normal controls met the criteria for cognitive impairment based on their MoCA score $(<26)$. In controls, CLOX1 and CLOX2 scores were $12.4 \pm 1.49$ and $13.8 \pm 1.2$, respectively, in accord with previous reports. The average administration time for the MoCA was $<15$ min. The order of administration did not affect test performance. MMSE, MoCA, CLOX1 and CLOX2 scores were gradually decreasing from controls to mild and moderate VaD patients, as expected (table 1).

ROC analyses for mild VaD versus controls, and mild versus moderate $\mathrm{VaD}$ are shown in table 2. In control and mild dementia groups, a cutoff score $\leq 21.5$ on the MoCA resulted in excellent sensitivity (86.8\%) and specificity (92.9\%) for detecting mild VaD (area under the curve, $\mathrm{AUC}=0.934$; fig. 1 ). In the patients with mild or moderate $\mathrm{VaD}$, a score $\leq 13.5$ was optimal for the MoCA to detect moderate dementia, with a sensitivity of $81.0 \%$ and specificity of $85.7 \%(\mathrm{AUC}=0.857)$.

\section{Discussion}

Brief screening instruments provide an objective and cost-effective means of determining the need for further evaluation of cognitive problems for older individuals at risk for dementia, if they are easy to administer, score, and interpret, and have acceptable ability to identify dementia or MCI [10]. This study provides data showing that the CC-MoCA, a 30-point scale including memory, attention, and executive and language tasks, is effective in differentiating SIVD patients from non-stroke, normal-cognition controls, and has also demonstrated good concurrent validity, as shown by the significant correlations with the MMSE and CLOX test. Consistent with previous reports, we found that performance on the CC-MoCA is affected by education [19-21], but not by age or gender. As stated by Lee et al. 
Fig. 1. ROC curve depicting the ability of the CC-MoCA in differentiating patients with mild SIVD from controls. AUC = $0.934, \mathrm{p}<0.001$.

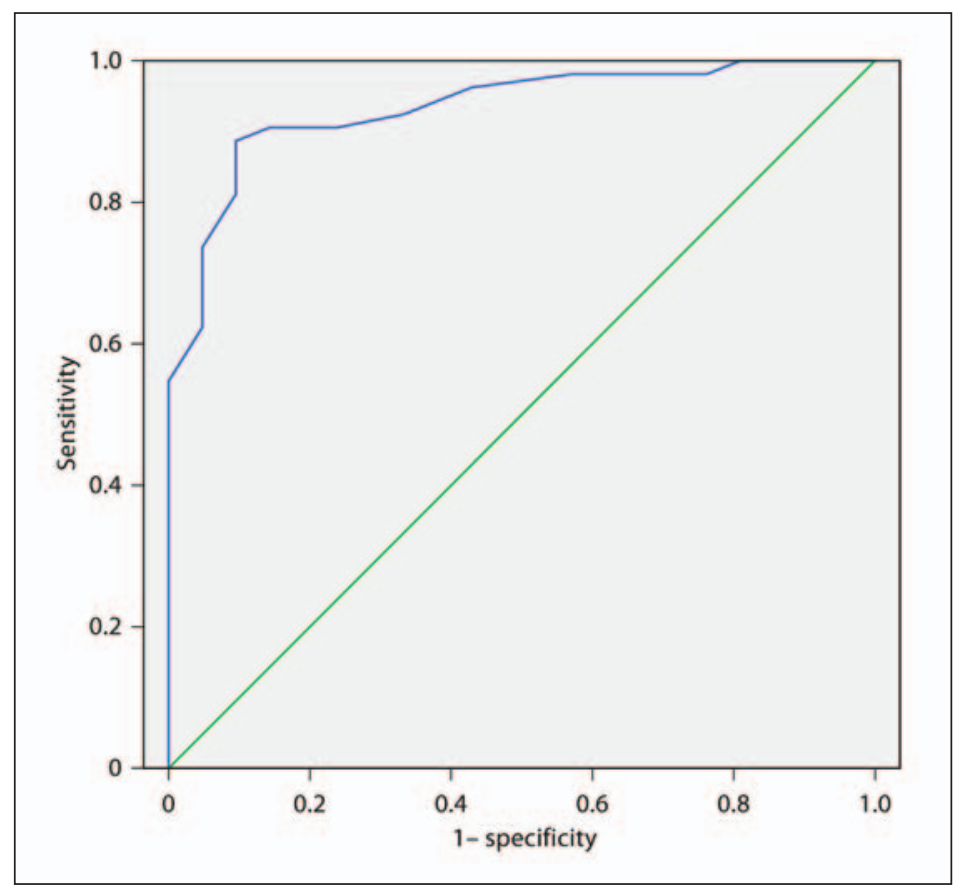

Table 2. ROC analyses for mild VaD versus controls, and mild versus moderate VaD

\begin{tabular}{|c|c|c|c|c|}
\hline Test parameter & AUC & Cutoff & Sensitivity & Specificity \\
\hline \multicolumn{5}{|c|}{ Mild VaD vs. controls } \\
\hline MMSE & 0.991 & 26.5 & 0.918 & 1.000 \\
\hline MoCA & 0.934 & 21.5 & 0.868 & 0.929 \\
\hline CLOX1 & 0.831 & 11.5 & 0.912 & 0.633 \\
\hline CLOX2 & 0.756 & 12.5 & 0.825 & 0.633 \\
\hline \multicolumn{5}{|c|}{ Mild vs. moderate VaD } \\
\hline MMSE & 1.000 & 20.5 & 0.933 & 1.000 \\
\hline MoCA & 0.857 & 13.5 & 0.810 & 0.857 \\
\hline CLOX1 & 0.721 & 7.5 & 0.700 & 0.559 \\
\hline CLOX2 & 0.793 & 10.5 & 0.867 & 0.636 \\
\hline
\end{tabular}

Relationship between scales: the scores of MoCA correlated significantly with that of MMSE $(r=0.886$, $\mathrm{p}<0.001)$, CLOX1 $(\mathrm{r}=0.673, \mathrm{p}<0.001)$ and CLOX2 $(\mathrm{r}=0.613, \mathrm{p}<0.001)$ in all subjects.

[13] and Wong et al. [19], in many Asian countries, elderly received much less education than their counterparts in Western countries. The average education level of our Chinese sample is 7-10 years, which is lower than the 12 years of education reported in Caucasian studies [5]. An education cutoff at 12 years, as proposed in the original MoCA study, is therefore not useful given that only $16 \%$ of our subjects were above this cutoff.

For all subjects, the scores on the CC-MoCA were significantly lower than the scores for the MMSE. Memory testing using the MoCA involves more words and a longer delay than in the MMSE. In contrast to the MMSE, the MoCA also assesses domains affected early in $\mathrm{VaD}$ patients, e.g. executive and visuospatial functions. ROC analysis suggested that CCMoCA provided both excellent sensitivity and specificity for discriminating mild VaD pa- 
tients from healthy controls at a cutoff value 21/22, and for discriminating moderate from mild VaD patients at a cutoff value 13/14. However, we also found that the MMSE gave higher values of AUC, sensitivity and specificity than CC-MoCA. However, since the MoCA was primarily developed and validated to detect VCI in particular, there may be a floor effect in mild/moderate VaD patients.

The correlation between the MMSE and the CC-MoCA was moderately high, in agreement with other studies $[9,10,19]$. The CLOX test was selected to help discriminate the executive control of clock drawing from clock drawing itself. The difference between CLOX1 and CLOX2 is hypothesized to reflect the specific contribution of executive control versus visuospatial tasks to overall clock drawing performance assessed by CLOX1 [18]. The relatively higher scores in CLOX2 than CLOX1 in our VaD patients were reflective of the prominent executive function impairments in SIVD.

There are several limitations of this study. First, we used a limited cognitive testing battery. Second, we only examined the ability of the CC-MoCA to differentiate between VaD patients and controls, rather than between patients with VCI with and without dementia. Unfortunately, the small sample size does not allow further stratification of patients into cognitive subgroups. Future studies including a larger sample size should be designed for this purpose, and patients with VCI without dementia should be recruited. Last, in our study, some SIVD patients possibly had concurrent $\mathrm{AD}$, because the clinical diagnosis is not the gold standard. In future studies, analysis of the concentrations of three protein biomarkers in cerebrospinal fluid (amyloid $\beta_{1-42}$, total tau and phosphorylated tau) is required to indicate the presence of $\mathrm{AD}$.

In conclusion, the CC-MoCA is a psychometrically valid, reliable and clinically useful screening test for patients with SIVD.

\section{References}

1 Moorhouse P, Rockwood K: Vascular cognitive impairment: current concepts and clinical developments. Lancet Neurol 2008;7:246-255.

-2 Chui HC: Subcortical ischemic vascular dementia. Neurol Clin 2007;25:717-740.

-3 Folstein MF, Folstein SE, McHugh PR: 'Mini mental state'. A practical method for grading the cognitive state of patients for clinicians. J Psychiatr Res 1975;12:189-198.

-4 Srikanth V, Thrift AG, Fryer JL, Saling MM, Dewey HM, Sturm JW, Donnan GA: The validity of brief screening cognitive instruments in the diagnosis of cognitive impairment and dementia after first-ever stroke. Int Psychogeriatr 2006;18:295-305.

-5 Nasreddine ZS, Phillips NA, Bedirian V, Charbonneau S, Whitehead V, Collin I, Cummings JL, Chertkow H: The Montreal Cognitive Assessment, MoCA: a brief screening tool for mild cognitive impairment. J Am Geriatr Soc 2005;53:695-699.

-6 Hachinski V, Iadecola C, Petersen RC, Breteler MM, Nyenhuis DL, Black SE, Powers WJ, DeCarli C, Merino JG, Kalaria RN, Vinters HV, Holtzman DM, Rosenberg GA, Wallin A, Dichgans M, Marler JR, Leblanc GG: National Institute of Neurological Disorders and Stroke-Canadian Stroke Network vascular cognitive impairment harmonization standards. Stroke 2006;37:2220-2241.

-7 Smith T, Gildeh N, Holmes C: The Montreal Cognitive Assessment: validity and utility in a memory clinic setting. Can J Psychiatry 2007;52:329-332.

$\rightarrow 8$ Lee JY, Dong Woo L, Cho SJ, Na DL, Hong Jin Jeon, Kim SK, You Ra Lee, Youn JH, Kwon M, Lee JH, Maeng Je Cho: Brief screening for mild cognitive impairment in elderly outpatient clinic: validation of the Korean version of the Montreal Cognitive Assessment. J Geriatr Psychiatry Neurol 2008;21: $104-110$.

-9 Zadikoff C, Fox SH, Tang-Wai DF, Thomsen T, de Bie RM, Wadia P, Miyasaki J, Duff-Canning S, Lang AE, Marras C: A comparison of the mini mental state exam to the Montreal Cognitive Assessment in identifying cognitive deficits in Parkinson's disease. Mov Disord 2008;23:297-299. 
-10 Luis CA, Keegan AP, Mullan M: Cross validation of the Montreal Cognitive Assessment in community dwelling older adults residing in the Southeastern US. Int J Geriatr Psychiatry 2009;24:197-201.

-11 Dalrymple-Alford JC, MacAskill MR, Nakas CT, Livingston L, Graham C, Crucian GP, Melzer TR, Kirwan J, Keenan R, Wells S, Porter RJ, Watts R, Anderson TJ: The MoCA: well-suited screen for cognitive impairment in Parkinson disease. Neurology 2010;75:1717-1725.

-12 Smith T, Gildeh N, Holmes C: The Montreal Cognitive Assessment: validity and utility in a memory clinic setting. Can J Psychiatry 2007;52:329-332.

-13 Lee Y, Dong Woo L, Cho S, Na DL, Hong Jin Jeon, Kim SK, You Ra Lee, Youn JH, Kwon M, Lee JH, Maeng Je Cho: Brief screening for mild cognitive impairment in elderly outpatient clinic: validation of the Korean version of the Montreal Cognitive Assessment. J Geriatr Psychiatry Neurol 2008;21: 104-110.

-14 Hughes CP, Berg L, Danziger WL, Coben LA, Martin RL: A new clinical scale for the staging of dementia. Br J Psychiatry 1982;140:566-572.

15 American Psychological Association: Diagnostic and Statistical Manual of Mental Disorders, ed 4. Washington, American Psychiatric Association, 1994.

-16 Wahlund LO, Barkhof F, Fazekas F, Bronge L, Augustin M, Sjogren M, Wallin A, Ader H, Leys D, Pantoni L, Pasquier F, Erkinjuntti T, Scheltens P: A new rating scale for age-related white matter changes applicable to MRI and CT. Stroke 2001;32:1318-1322.

17 Chiu HFK, Kee HC, Chung WS, Kwong PK: Reliability and validity of the Cantonese version of MiniMental State Examination - a preliminary study. J Hong Kong Coll Psychiatry 1994;4:25-28.

-18 Royall DR, Cordes JA, Polk M: CLOX: an executive clock drawing task. J Neurol Neurosurg Psychiatry 1998;64:588-594.

-19 Wong A, Xiong YY, Kwan PWL, Chan AY, Lam WW, Wang K, Chu WC, Nyenhuis DL, Nasreddine Z, Wong LK, Mok VC: The validity, reliability and clinical utility of the Hong Kong Montreal Cognitive Assessment (HK-MoCA) in patients with cerebral small vessel disease. Dement Geriatr Cogn Disord 2009;28:81-87.

-20 Wen HB, Zhang ZX, Niu FS, Li L: The application of Montreal Cognitive Assessment in urban Chinese residents of Beijing (in Chinese). Zhonghua Nei Ke Za Zhi 2008;47:36-39.

-21 Wang RL, Qu QM, Cao HM, Zhe X, Qiao J, Guo F: Study on the status of cognition among Parkinson's disease patients in Xi'an (in Chinese). Zhonghua Liu Xing Bing Xue Za Zhi 2010;31:329-332. 